Table 1. Estimation of the model parameter $p$ using linear regression analyses for

$$
\left(\ln \dot{\varepsilon}_{c}-\ln \dot{\varepsilon}_{0}\right) \text { and }-\ln (1-\omega) \text {. }
$$

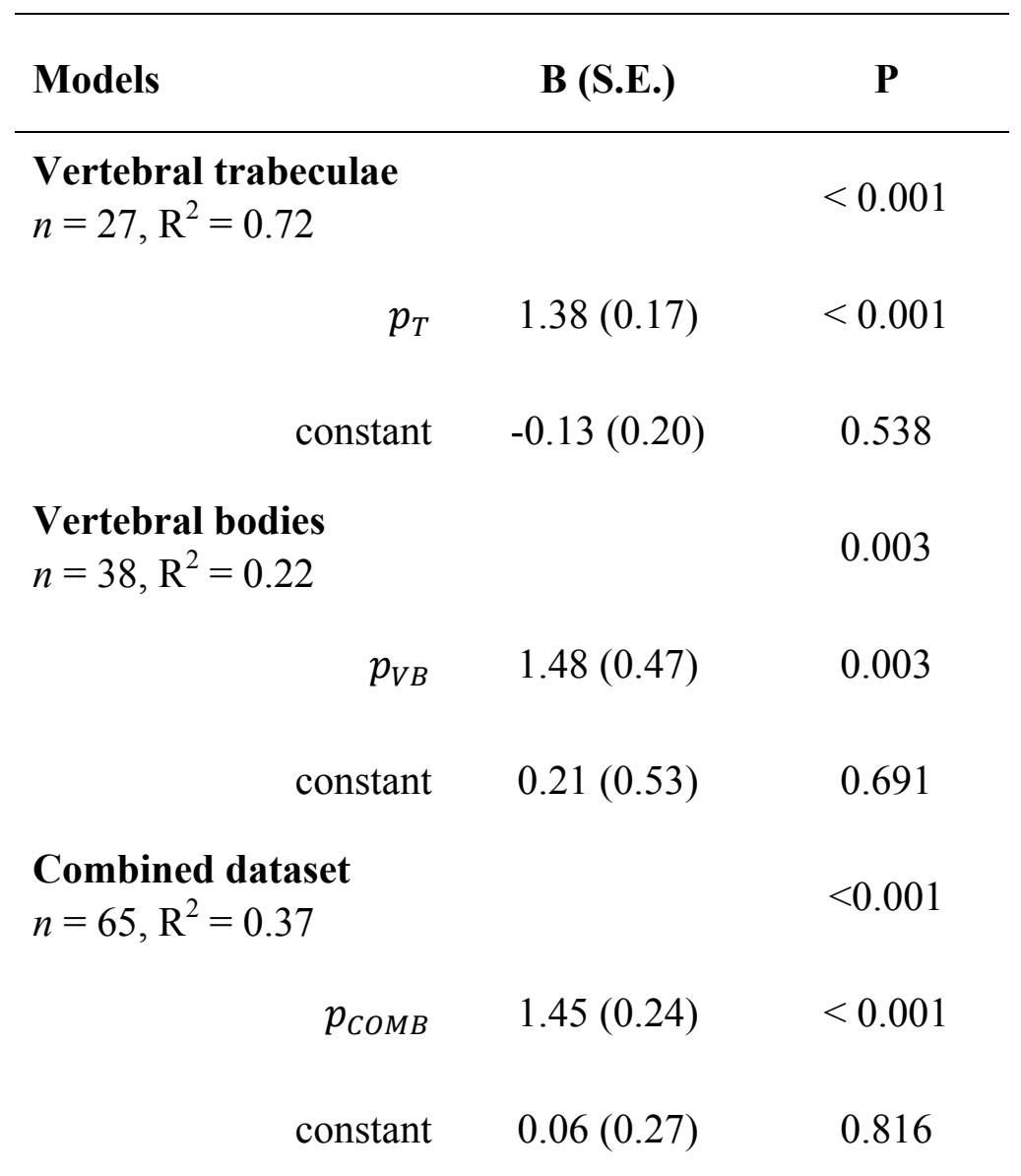

Equation 5 in the text was used. $p_{T}, p_{V B}$, and $p_{\text {СОMB }}$ : model parameter estimated from mechanical data of vertebral trabeculae samples, vertebral bodies, and combined data, respectively. $n$ : number of specimens tested; B: unstandardized regression coefficient; S.E. $=$ standard error 
Table 2. Results from regression analyses examining the moderation of endplate damage, cortical bone damage, disc degeneration, vertebral cross-sectional area (CSA), and vertebral aBMD on the model parameter $p_{V B} \quad(n=38)$

\section{Moderation analysis}

B (S.E.)

\section{$\mathbf{P}$}

Model 1: moderation effect of endplate damage

$\mathrm{R}^{2}=0.24$

$$
-\ln (1-\omega)\left(p_{V B}\right)
$$

Endplate damage $\left(b_{M}\right)$

$-0.14(0.49)$

0.048

Endplate damage $\times[-\ln (1-\omega)]\left(b_{I}\right)$

0.776

Model 2: moderation effect of cortical bone damage

$\mathrm{R}^{2}=0.26$

$$
-\ln (1-\omega)\left(p_{V B}\right)
$$

Cortical bone damage $\left(b_{M}\right)$

Cortical bone damage $\times[-\ln (1-\omega)]\left(b_{I}\right)$

Model 3: moderation effect of disc degeneration $\mathrm{R}^{2}=0.28$

$$
-\ln (1-\omega)\left(p_{V B}\right)
$$

Disc degeneration $\left(b_{M}\right)$

Disc degeneration $\times[-\ln (1-\omega)]\left(b_{I}\right)$

Model 4: moderation effect of vertebral CSA

$\mathrm{R}^{2}=0.24$

$$
-\ln (1-\omega)\left(p_{V B}\right)
$$

0.008

Cross-sectional area $\left(b_{M}\right)$

Cross-sectional area $\times[-\ln (1-\omega)]\left(b_{I}\right)$

Model 5: moderation effect of vertebral aBMD $\mathrm{R}^{2}=0.28$

$$
-\ln (1-\omega)\left(p_{V B}\right)
$$

Vertebral aBMD $\left(b_{M}\right)$

Vertebral aBMD $\times[-\ln (1-\omega)]\left(b_{I}\right)$

$n=$ number of specimens; $\mathrm{B}=$ unstandardized coefficient; S.E. $=$ standard error. 